\title{
Erratum: Parametrized post-Einsteinian gravitational waveforms in various modified theories of gravity [Phys. Rev. D 98, 084042 (2018)]
}

\author{
Sharaban Tahura $\odot$ and Kent Yagi
}

(Received 29 December 2019; published 27 May 2020)

DOI: $10.1103 /$ PhysRevD.101.109902

An error was found in our earlier Letter [1], which corrects Eq. (32) of this paper [2] as

$$
\gamma_{\dot{f}}=\frac{11975}{12288} \eta^{-9 / 5} \zeta_{\mathrm{dCS}} \chi_{1} \chi_{2}-\frac{96305}{172032} \eta^{-14 / 5} \zeta_{\mathrm{dCS}}\left(\frac{m_{1}^{2}}{m^{2}} \chi_{2}^{2}+\frac{m_{2}^{2}}{m^{2}} \chi_{1}^{2}\right)
$$

This further corrects Eqs. (33) and (34) of this paper [2] as

$$
\alpha_{\mathrm{dCS}}=\frac{57713}{344064} \eta^{-14 / 5} \zeta_{\mathrm{dCS}}\left[-2 \delta_{m} \chi_{a} \chi_{s}+\left(1-\frac{14976 \eta}{57713}\right) \chi_{a}^{2}+\left(1-\frac{215876 \eta}{57713}\right) \chi_{s}^{2}\right]
$$

TABLE I. PPE corrections to the GW phase $\delta \Psi \equiv \beta u^{b}$ in Fourier space in various modified theories of gravity, where $\beta$ is the magnitude correction (second column) and $b$ is the exponent correction (third column). $u \equiv\left(\pi G_{C} \mathcal{M} f\right)^{1 / 3}$, where $\mathcal{M}$ and $\eta$ are the chirp mass and the symmetric mass ratio of the binary respectively, and $G_{C}$ is the conservative gravitational constant appearing in Kepler's third law. We adopt the unit $G_{C} \equiv 1$ in all theories except for the varying- $G$ ones. The expressions in dynamical Chern-Simons (dCS) gravity and noncommutative gravity only apply to binary BHs, while those in other theories apply to any compact binaries (last column) ${ }^{\text {a }}$. The mass, sensitivity, and scalar charge of the Ath binary component are represented by $m_{A}, s_{A}$, and $\alpha_{A}$ respectively. $\zeta_{\mathrm{EdGB}}$ and $\zeta_{\mathrm{dCS}}$ are the dimensionless coupling constants in Einstein-dilaton Gauss-Bonnet (EdGB) and dCS gravity respectively. $\tilde{s}_{A}^{\text {EdGB }}$ are the spin-dependent factors of the scalar charges in EdGB gravity, given below Eq. (29) of [2] for BHs while 0 for ordinary stars. $\chi_{s, a}$ are the symmetric and antisymmetric combinations of dimensionless spin parameters and $\delta_{m}$ is the fractional difference in masses relative to the total mass $m$. The amount of Lorentz violation in Einstein-Æther theory and khronometric gravity is controlled by $\left(c_{1}, c_{2}, c_{3}, c_{4}\right)$ and $\left(\bar{\alpha}_{\mathrm{kh}}, \bar{\beta}_{\mathrm{kh}}, \bar{\lambda}_{\mathrm{kh}}\right)$ respectively. $w_{s}$ is the propagation speed of the spin-s modes in Einstein-Æther theory given by Eqs. (37)-(39) of [2], and $c_{14} \equiv c_{1}+c_{4}$. The representative parameter in noncommutative gravity is $\Lambda$. The subscript 0 in varying- $G$ theories denotes that the quantity is measured at the time of coalescence $t_{0}$, while a dot refers to a time derivative. $\delta_{\dot{G}}$ is the fractional difference between the rates at which conservative and dissipative gravitational constants change in time. The former is $G_{C}$ as already explained while the dissipative gravitational constant is defined as the one that enters in the GW luminosity through Eq. (8) of the [2]. The boldface expression indicates

\begin{tabular}{|c|c|c|c|}
\hline \multirow[t]{2}{*}{ Theories } & \multicolumn{2}{|l|}{ PPE phase parameters } & \multirow[t]{2}{*}{ Binary type } \\
\hline & Magnitude $(\beta)$ & Exponent $(b)$ & \\
\hline Scalar-tensor $[4,5]$ & $-\frac{5}{7168} \eta^{2 / 5}\left(\alpha_{1}-\alpha_{2}\right)^{2}$ & -7 & Any \\
\hline EdGB [6] & $-\frac{5}{7168} \zeta_{\mathrm{EdGB}} \frac{\left(m_{1}^{2} \tilde{S}_{2}^{\mathrm{EdGB}}-m_{2}^{2} \tilde{S}_{1}^{\mathrm{ddGB}}\right)^{2}}{m^{4} \eta^{18 / 5}}$ & -7 & Any \\
\hline $\operatorname{DCS}[1,7]$ & $\frac{481525}{3670016} \eta^{-14 / 5} \zeta_{\mathrm{dCS}}\left[-2 \delta_{m} \chi_{a} \chi_{s}+\left(1-\frac{4992 \eta}{19261}\right) \chi_{a}^{2}+\left(1-\frac{72052 \eta}{19261}\right) \chi_{s}^{2}\right]$ & -1 & $\mathrm{BH} / \mathrm{BH}$ \\
\hline Einstein-Æther [8] & $-\frac{5}{3584} \eta^{2 / 5} \frac{\left(s_{1}^{\mathrm{EA}}-s_{2}^{\mathrm{EA}}\right)^{2}}{\left[\left(1-s_{1}^{\mathrm{EA}}\right)\left(1-s_{2}^{\mathrm{EA}}\right)\right]^{4 / 3}}\left[\frac{\left(c_{14}-2\right) w_{0}^{3}-w_{1}^{3}}{c_{14} w_{0}^{3} w_{1}^{3}}\right]$ & -7 & Any \\
\hline Khronometric [8] & $-\frac{5}{3584} \eta^{2 / 5} \frac{\left(s_{1}^{\mathrm{kh}}-s_{2}^{\mathrm{kh}}\right)^{2}}{\left[\left(1-s_{1}^{\mathrm{kh}}\right)\left(1-s_{2}^{\mathrm{kh}}\right)\right]^{4 / 3}} \sqrt{\bar{\alpha}_{\mathrm{kh}}}\left[\frac{\left(\bar{\beta}_{\mathrm{kh}}-1\right)\left(2+\bar{\beta}_{\mathrm{kh}}+3 \bar{\lambda}_{\mathrm{kh}}\right)}{\left(\bar{\alpha}_{\mathrm{kh}}-2\right)\left(\bar{\beta}_{\mathrm{kh}}+\bar{\lambda}_{\mathrm{kh}}\right)}\right]^{3 / 2}$ & -7 & Any \\
\hline Noncommutative [9] & $-\frac{75}{256} \eta^{-4 / 5}(2 \eta-1) \Lambda^{2}$ & -1 & $\mathrm{BH} / \mathrm{BH}$ \\
\hline Varying- $G[10]$ & $-\frac{25}{851968} \eta_{0}^{3 / 5} \dot{\mathbf{G}}_{\mathbf{C}, \mathbf{0}}\left[11 \mathbf{m}_{\mathbf{0}}+\mathbf{3}\left(\mathbf{s}_{1,0}+\mathbf{s}_{2,0}-\delta_{\dot{G}}\right) \mathbf{m}_{\mathbf{0}}-\mathbf{4 1}\left(\mathbf{m}_{1,0} \mathbf{s}_{1,0}+\mathbf{m}_{2,0} \mathbf{s}_{2,0}\right)\right]$ & -13 & Any \\
\hline
\end{tabular}
that it has been derived in this paper [2] for the first time.

${ }^{\mathrm{a}}$ Practically speaking, if NSs are spinning much slower than BHs, one can use the dCS expression also for BH/NS binaries by setting one of the spins to zero. 
TABLE II. PPE corrections to the GW amplitude $|\tilde{h}|=\left|\tilde{h}_{\mathrm{GR}}\right|\left(1+\alpha u^{a}\right)$ in Fourier space in various modified theories of gravity with the magnitude $\alpha$ (second column) and the exponent $a$ (third column), and $\left|\tilde{h}_{\mathrm{GR}}\right|$ representing the amplitude in GR. The meaning of other parameters are the same as in Table II. The expressions in boldface correspond to either those derived in [2] for the first time or corrected expressions from previous literature.

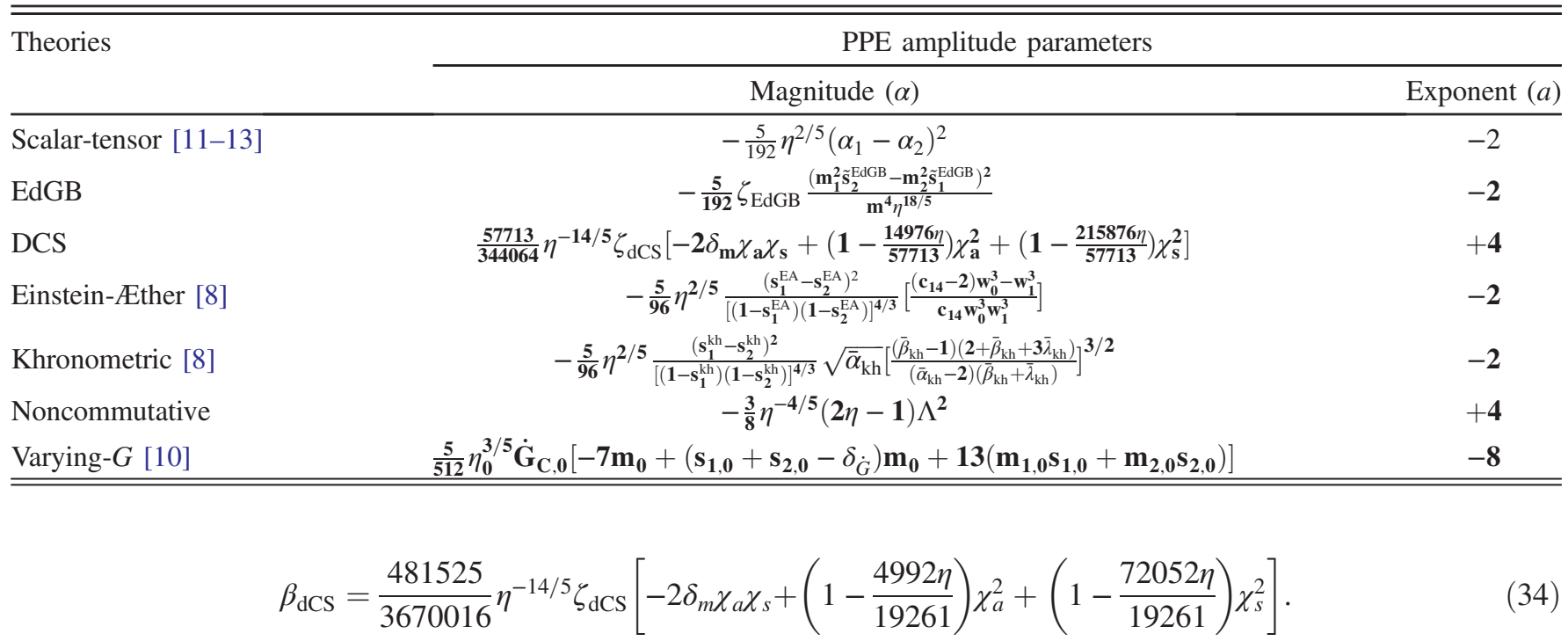

Equation (34) agrees with Eq. (3) of [3] to quadratic order in spin. The DCS rows in Tables I and II are corrected according to Eqs. (34) and (33) above respectively. We present the corrected tables in this erratum for completeness. Since we do not derive any constraint on the coupling parameter $\zeta_{\mathrm{dCS}}$, such corrections do not change any conclusion drawn on DCS theory in this paper.

[1] K. Yagi, N. Yunes, and T. Tanaka, Phys. Rev. Lett. 109, 251105 (2012); 116, 169902(E) (2016).

[2] S. Tahura and K. Yagi, Phys. Rev. D 98, 084042 (2018).

[3] R. Nair, S. Perkins, H. O. Silva, and N. Yunes, Phys. Rev. Lett. 123, 191101 (2019).

[4] P. D. Scharre and C. M. Will, Phys. Rev. D 65, 042002 (2002).

[5] E. Berti, A. Buonanno, and C. M. Will, Phys. Rev. D 71, 084025 (2005).

[6] K. Yagi, L. C. Stein, N. Yunes, and T. Tanaka, Phys. Rev. D 85, 064022 (2012); 93, 029902(E) (2016).

[7] N. Yunes, K. Yagi, and F. Pretorius, Phys. Rev. D 94, 084002 (2016).

[8] D. Hansen, N. Yunes, and K. Yagi, Phys. Rev. D 91, 082003 (2015).

[9] A. Kobakhidze, C. Lagger, and A. Manning, Phys. Rev. D 94, 064033 (2016).

[10] N. Yunes, F. Pretorius, and D. Spergel, Phys. Rev. D 81, 064018 (2010).

[11] K. G. Arun, Classical Quantum Gravity 29, 075011 (2012).

[12] K. Chatziioannou, N. Yunes, and N. Cornish, Phys. Rev. D 86, 022004 (2012); 95, 129901(E) (2017).

[13] T. Liu, X. Zhang, W. Zhao, K. Lin, C. Zhang, S. Zhang, X. Zhao, T. Zhu, and A. Wang, Phys. Rev. D 98, 083023 (2018). 